\title{
La psicología social en América latina: Desarrollo y tendencias actuales
}

\author{
MARITZA MONTERO \\ Universidad Central de Venezuela. Conferencia dictada \\ en la Universidad de Costa Rica, el 26-5-1986
}

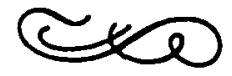

La Psicología social, como muchas otras ramas de la Psicología, tiene un desarrollo relativamente reciente en nuestra parte del continente. De hecho, muchas cátedras de Psicología Social sólo vienen a ser creadas a fines de la década del cincuenta o a principios de los años sesenta (En Brasil hacia 1953; en Venezuela en 1954 y en Chile en 1962). Esto supone por una parte, que quienes originaron y regentaron esas cátedras, necesariamente eran personas formadas en contextos académicos no latinoamericanos, es decir: europeos y norteamericanos, donde la disciplina tenía ya varias décadas de desarrollo. Y por otra, que hubo de transcurrir un cierto tiempo antes de que la enseñanza dictada en esas cátedras dejase de ser un mero aditamento curricular o el complemento de una formación que creemos que en sus orígenes estuvo fundamentalmente dirigida hacia la produccción de psicólogos clínicos y orientadores, para pasar a generar un campo de trabajo propio, intereses específicos y productos directamente ligados a un quehacer independiente, en tanto que rama de la Psicología.

Y ello se explica porqué la Psicología es, en el contexto de las ciencias sociales, una de las más recientes en cuanto a organización sistemática, a generación de métodos y lenguaje propios y a la determinación clara de su objeto de estudio. Si bien pueden hallarse reflexiones de índole psicológica en muchos filósofos a lo largo de la historia, en sentido estricto podemos decir que es a partir de la creación del laboratorio de W. Wundt en 1879, en Leizpig, cuando la Psicología se inicia como ciencia per se. Y en cuanto a la psicología social cabe hacer la misma observación: numerosas reflexiones en los campos de la filosofía, de la antropología, de la sociología y de la misma psicología (un ejemplo es la obra de Wundt, Psicología de los Pueblos), se acercaron a ella, pero no es sino hasta 1908, con los trabajos simultáneos de un psicólogo, McDougall y de un sociólogo, Ross, cuando va a surgir como rama específica de esa ciencia. Interesante orígen que marca ya su destino interdisciplinario.

Su desarrollo en Norteamérica y en Europa no pasa desapercibido para América Latina, pero es incorporado como parte de las disquisiciones sociológicas, sociopolíticas o antropológicas (cf. Arthur Ramos: Introducción 
a la Psicología Social, Rio de Janeiro, 1956), y no como un cuerpo de conocimientos que debiese ser desarrollado aparte. Así, podemos encontrar que en algunos autores de principios de siglo, preocupados por la problemática del "carácter nacional", de la identidad social; en fin, por definir nuestros pueblos, incluyeron en sus obras capítulos sobre la psicologia popular en sus respectivos países, o inclusive usaron la denominación de psicología social, pero siempre con ese mismo sentido.

Mientras tanto, otras ramas de la psicología van obteniendo desarrollos y logros, a veces espectaculares, que se dirigen a aspectos directamente evidentes de la realidad social e individual: la enfermedad mental; el desarrollo cognoscitivo; la productividad en el trabajo; la adaptación, progreso, retardo y en general problemas que puede presentar el individuo en su contacto con la institución escolar. Son las ramas de la psicología unidas a estos aspectos las que primero serán desarrolladas en los países latinoamericanos.

Pero la influencia social sobre individuo y grupo, los cambios conductuales que en ellos se operan en relación con cambios sociales, el substrato psicológico de ciertas conductas colectivas, no pueden ignorarse durante mucho tiempo, y ya para mediados de la década del sesenta, puede decirse que existe una clara conciencia de la necesidad de desarrollar en nuestros países una psicología social que ayude a la comprensión de la interacción individuo-grupo-sociedad. Así encontramos entre otros, los trabajos aislados de Rogelio Díaz Guerrero (1966) en México, en los cuales lo psicosocial está aún muy ligado a lo psicodinámico en sus aspectos clínicos; en Brasil, las réplicas hechas por Aroldo Rodrigues a los experimentos hechos en Norteamérica por los creadores de las teorias del equilibrio, así como sus estudios en la línea de Heider (1965; 1967, p.e.); en Venezuela los trabajos sobre estereotipos nacionales de Constancio De Castro (1968) y actitudes nacionalistas de J. M. Salazar (publicado en 1970, pero realizado con anterioridad).

Pero ya para la década del setenta, comienza a haber una producción más nutrida y continua. Así, en 1972 aparece el que creemos es el primer manual latinoamericano en la disciplina: Estudos em Psicologia Social, de Aroldo Rodrigues, editado en Petrópolis y luego, en castellano, en México (Trillas, 1975); seguido en 1976 por otro publicado primero por la Asociación Venezolana de Psicología social y la Escuela de Psicología de la Universidad Central de Venezuela, y luego por Trillas, en México (1978). Se trataba en este caso de una obra colectiva escrita por algunos miembros del Departamento de Psicología Social de la Universidad mencionada. A estas dos obras se une la recopilación de artículos sobre temas psicosociales escritos por autores latinoamericanos, hecha por Gerardo Marín y publicada en 1975 (La Psicología Social en Latinoamérica), a la cual seguirá un segundo volumen en 1981.

No seguiremos haciendo una descripción bibliográfica que haría pesado este recuento y no añadiría mucho al análisis que pretendemos desarrollar. Baste decir que a partir de 1972 la producción es evidente y que en lo que va de esta década, está además marcada ya por ciertos signos que tipifican un desarrollo original y que le comienzan a dar fisonomía propia.

En su evolución, así como en el vuelco hacia lo real que hace la Psico- 
logía Social latinoamericana, es necesario sin embargo destacar ciertos acontecimientos que consideramos relevantes:

En primer lugar la creación de una Asociación Latinoamericana de Psicología Social (ALAPSO), que surge como iniciativa de un grupo de psicólogos en varios países del area, a partir del Comité Latinoamericano de Psicología Social (con las fatídicas siglas de COLAPSO, que deben haber incidido en su rápida desaparición), y que recibe el auspicio económico de una casa editorial mexicana. Este grupo realizó en 1973 un Encuentro Latinoamericano de Psicología Social en Bogotá, Colombia, en el cual se sentaron las bases para la creación de la Asociación y de un futuro Colegio Latinoamericano de Psicología Social, con sede en México, que nunca llegó a cristalizar. Pero la ALAPSO adquirió vida y en 1975, con la colaboración de la Asociación Venezolana de Psicología Social (AVEPSO), entonces recien creada, se realizó el Primer Seminario Latinoamericano de Psicología Social, en Caracas, organizado por AVEPSO, el cual permitió establecer una serie de contactos y relaciones entre investigadores de diversos paises, que luego probaron ser particularmente fructíferas. Posteriormente ALAPSO realizó en México dos o tres seminarios más, y creó la $R e$ vista Latinoamericana de Psicologia Social, de la cual salieron hasta ahora cuatro números. Aunque actualmente tiene mucha menos proyección, en su momento constituyó un impulso para la Psicología Social, en la medida en que fue el elemento precipitante para el intercambio.

A su vez AVEPSO comenzó a publicar en 1977 un Boletín de aparición cuatrimestral, que circula en casi toda América Latina, los EEUU de Norteamérica y en algunos países de Europa, llegando sus resúmenes, actualmente, a tres mil instituciones en el mundo. Esto, más el respaldo editorial habido y la acogida recibida en las otras pocas publicaciones periódicas de nuestra parte del Continente (Revista Latinoamericana de Psicología, Revista Interamericana de Psicología, Estudios Centroamericanos, entre otras), han permitido a esta Psicología Social naciente, en busca de su definición, que lucha por construirse un ámbito, hacer oir su voz.

La evolución de la disciplina que nos concierne presenta, a nuestro parecer, dos etapas hasta ahora: una primera, de generación y estructuración, en la cual su principal tarea era conseguir un puesto académico y que prodríamos, en función de ello, denominar período o etapa del desarrollo académico. En ella, la psicología social tiene como tarea fundamental resumir el conocimiento producido hasta el momento en los EEUU de Norteamérica y en Europa, aplicando métodos y técnicas surgidos en esas latitudes, a problemas a menudo copiados de los reseñados en las revistas científicas especializadas, o derivadas de problemas que las teorías estudiadas presentan. Los psicólogos sociales se preguntan en esta etapa: ¿se aplicarán aquí, en este país, en estas condiciones, esas leyes? ¿operan los mismos principios?, o bien, sin cuestionamiento, sin interrogante alguna, los ponen en práctica. Pero también surgen, no sin cierta timidez, problemas específicos, que aun estudiados bajo el prisma de las teorías provenientes de los centros irradiadores y con instrumentos creados para otras culturas, dan resultados que señalan irregularidades, peculiaridades, aspectos atípicos, que no pueden menos que preocupar a esos psicólogos sociales. ¿Fallas en la teoría? ¿fallas de los sujetos observados? ¿fallas del método? ¿fallas del investigador? Los mecanismos de control inherentes al método científico permiten 
ir descartando cada supuesto y poco a poco, una verdad se ha hecho palpable: Es necesario buscar otros métodos, otras explicaciones, o bien introducir modificaciones a las existentes (Montero, 1976). Es ese el momento en que comienza a surgir una ciencia propia. Un aporte original latinoamericano a la psicología social.

Resumiendo, esta primera etapa se caracteriza por la sistematización académica, la aplicación predominante del método experimental, el uso de técnicas e instrumentos transplantados directamente de los países donde fueron originados y trabajos en los cuales, fundamentalmente, se explora, describe y diagnostica.

Una segunda etapa surge aproximadamente a partir de los años setenta, coexistiendo con remanentes de la anterior. En este período hay una evolución hacia la definición de la Piscología Social en términos de la problemática a la cual se dirige su estudio, y de la intervención sobre la misma. Es la etapa de la autodefinición y adecuación a realidades sociales específicas. Un aporte a esa definición es enriquecer su objeto de estudio con el campo de la ideología (Salazar, 1976; 1983; Montero, 1984; Martín-Baró, 1983), algo en lo que nuestra América ha tomado la delantera a otras regiones del mundo.

Esta etapa se caracteriza por la formulación de problemas que cada vez más, en lugar de provenir de las teorías o de otras realidades, surgen del ámbito en que se desenvuelven los investigadores, son planteados por la sociedad en que viven y responden a necesidades perentorias de su población. Se caracteriza además por la aplicación. Si antes se buscaba saber qué hacía, qué era la psicología social, ahora que se la ha redefinido, se quiere saber para qué sirve y se busca obtener resultados concretos que modifiquen la realidad. En este sentido, Ziviani en Brasil (1978) señala cómo comienza a desarrollarse en su país una psicología social que busca hacer contribuciones socialmente relevantes y emplear sus hallazgos para resolver problemas de la vida diaria.

Así es interesante observar cómo evoluciona esa necesidad. Ya desde 1972, los trabajos de Jacobo Varela exhortaban a los psicólogos sociales a crear, como en otras ciencias, una tecnología, que obviamente, en este caso sería social. Para ello recomendaba emplear múltiples hallazgos provenientes de nuestra propia ciencia, así como de otras, aparentemente aislados, combinándolos para dar soluciones novedosas a los problemas. Un enfoque que Varela ilustraba haciendo una conjunción de elementos provenientes de la teoría de la disonancia cognoscitiva, de la teoría de la reactancia psicológica, de los aportes de psicólogos como Stanley Schachter, Sherif, Deutsch y otros; de la teoría de los grupos y en general, de todas las corrientes vigentes en Norteamérica. Sin embargo, adelantada ya la década, encontramos que otro psicólogo latinoamericano, J. M. Salazar (1981), critica esta posición diciendo que la psicologia social aplicada puede ser concebida de dos maneras: «.. una [que] se centra sobre la aplicación de principios y la otra sobre el análisis y solución de problemas. Lo primero se aproxima a una tecnología; lo segundo, a la aplicación del método científico para dilucidar un problema que surge no del desarrollo de una teoría sino de la vida contidiana». Y a continuación se cuestionaba acerca de la factibilidad de una tecnología en psicología social, ya que tal cosa supone la existencia de principios universales, acerca de lo cual, aparte de una gran discusión, no hay todavía respuesta. 
Sánchez y Wiesenfeld (1982), haciendo un recuento de la posición lationoamericana al respecto, encuentran tres tendencias: 1) Considerar que la validez externa de las teorías de nuestra disciplina se limita a la cultura en que fueron producidas, por lo cual su generalización a otros contextos exige replicar las investigaciones que les dieron origen. Esta es la posición que ha sostenido en Brasil, Aroldo Rodrigues. 2) Producir nuevos modelos en América Latina relevantes para su realidad, partiendo de los hallazgos psicosociales como hipótesis, posición en la que destacan el panameño Luis A. Escovar y el mexicano Emilio Ribes Iñesta. 3) Creer que los hallazgos propios de la psicología social pueden contribuir a solucionar problemas sociales en diferentes contextos; posición en la cual estaría ubicado Varela, para quien el conocimiento sería universal.

Vemos así cómo evolucionan las consideraciones acerca de la aplicación de la psicología social, tendiendo a responder cada vez más a las necesidades de la sociedad, asumiendo incluso un carácter francamente motivado por la independencia y el deseo de generar respuestas originales.

Un examen aún superficial, de la producción psicosocial latinoamericana del setenta en adelante, nos muestra que si bien el transplante y seguimiento dócil de las corrientes teóricas generadas en los centros de poder no han dejado de existir, la segunda tendencia antes señalada, es la dominante no sólo en el campo de la aplicación, sino también en el de la generación de teoría y metodología. Así, la psicología social en el breve lapso de diez años (1970-1980), ha dado lugar al surgimiento simultáneo en varios países latinoamericanos, de una rama comunitaria (Psicología social comunitaria o psicología comunitaria o desarrollo de comunidades, entre otras denominaciones más o menos parciales). Efectivamente, de manera aislada pero coincidente, investigadores dominicanos, venezolanos, puertorriqueños, salvadoreños, mexicanos, peruanos, colombianos y brasileños, comprendimos que la psicología social podía ser un instrumento para el cambio social, que el psicólogo podía ser un catalizador o agente de ese cambio y que nuestra disiciplina adquiría una nueva dimensión y sentido, cuando, abandonando el recinto académico, se proyectaba hacia la comunidad.

Igualmente, otro brote del tronco psicosocial han sido los estudios sobre problemas ambientales, muchas veces estrechamente ligados a lo anterior. Y lo mismo puede decirse del campo político, que de tímidos diagnósticos acerca de actitudes o conductas específicas, ha pasado a formular una crítica psicosocial de la situación latinoamericana y comienza ahora a producir disquisiciones de orden teórico. Otra área de interés actual es la sexualidad como fenómeno social y la definición y atribuciones socialmente establecidos sobre los roles sexuales. Igualmente la relación salud-enfermedad como vía de sintomatización social; o la conducta desviada como expresión de la dinámica social. Y subyaciendo a todo ello, algo que hace el fondo común a todas estas áreas de investigación: la necesidad de responder a las exigencias de la realidad social que es única para cada país, pero que a la vez compartimos en toda América Latina en la medida en que poseemos rasgos culturales comunes y una posición periférica, mediatizada desde el punto de vista económico y político.

Según Luis Escovar (1980), en nuestra América coexisten cuatro tendencias: una psicología social tradicional, una piscología social aplicada, otra volcada hacia el desarrollo social y económico y finalmente, una de ca- 
rácter comunitario. Sin embargo, esta clasificación no es excluyente, de hecho la tendencia general es hacer psicología aplicada (tradicional o no) punto en el cual coinciden otros autores (Ribeiro de Almeida, sf; Pulido de Briceño, 1985, entre varios), y la psicología social comunitaria es planteada en muchos casos como una alternativa de desarrollo.

Puede decirse pues, que la psicología social en América Latina busca actualmente: 1) Librarse de la dependencia teórica y metodológica. 2) Forjarse una clara identidad científica. 3) Dar una respuesta a los problemas sociales de los países en que es aplicada, buscando a la vez definirse a sí misma. 4) Proyectarse en una perspectiva histórica y dinámica; algo que planteábamos ya en 1978 y a lo que tratamos de dar una base empírica en 1984, a la vez que en Brasil, Ana Jurema escribia sobre el tema (1985), y ya en 1972, desde otro ángulo, Díaz Guerrero en México, buscaba formular una teoria del comportamiento humano que respondiese a elementos históricos, biológicos, psicológicos, sociales y culturales. En El Salvador, los trabajos de Martín-Baró han dado un viraje liberador a la psicología social y en el mismo sentido se produce el libro de Silvia Lane y colaboradores en la Universidad de São Paulo, Brasil (1984), o los trabajos de J. Gissi y de Verro en Chile. Se define y se redefine, se aplica y se denuncia, sin por ello renegar de la acumulación teórica y metodológica previamente existente.

Si algo puede caracterizar a la psicología social latinoamericana actual, es por una parte la revisión crítica de la teoría, y por otra, su carácter interventor de la realidad, sobre la que actúa y el cual ella misma evalúa cada vez. La experimentación de laboratorio cede lugar a la de campo y aún más a la cuasi experimentación; comienzan a crearse técnicas, nuevas estrategias y métodos alternativos más acordes con la realidad a estudiar y con los enfoques a utilizar. Un ejemplo de esto es la posición sostenida por Escovar (1980), en la cual afirma que el psicólogo social para lograr cambios sociales, debe actuar sobre los sentimientos de alienación de la gente. Buscar ese nivel y atacarlo, a sabiendas de que no está dirigiendo su esfuerzo contra factores estructurales, fuera de su alcance profesional, ni volcándolo sobre conductas cuyas causas, normalmente alienantes, suelen permancer incólumes en el trabajo psicológico tradicional.

En cuanto a esa revisión crítica de la teoría, quizá la manifestación más reciente, es necesario decir que de una aceptación absoluta de las teorías nortemaericanas y europeas, se ha pasado a la aceptación matizada, en la cual predominan casi universalmente los enfoques cognitivistas, aunque no sin críticas. La mayoría de las investigaciones que se realizan versan sobre los procesos mediadores entre la realidad y el comportamiento social. Estos enfoques se han enriquecido con los aportes de la filosofía (teoría de la ideología), de la sociología y particularmente con los de la teoría de la dependencia y de la sociología comprometida, generadas en América Latina, así como con los desarrollos que a partir de las tesis de Marx han surgido en nuestro propio ambiente.

No es una tarea fácil. Una característica del subdesarrollo y de su condición periférica, es la ausencia de comunicaciones que no pasen por el Centro generador del conocimiento. Por ello hasta hace muy poco, y todavía hoy, hemos tenido un desconocimiento casi absoluto en el propio. A ello se ha unido la descalificación, que nuestra propia tendencia minusvaloran- 
te, ideológicamente manipulada, nos lleva muchas veces a arrojar sobre los trabajos que no provengan de los centros de desarrollo. No obstante, ya en muchos países del área, explícita o implícitamente, los psicólogos sociales han tomado conciencia de que el apelativo social no es un aditamento superfluo a la cualidad de psicólogo, sino que es un imperativo ligado a las condiciones de vida sobre las que va a actuar; que ejercer una psicología social significa un compromiso y que ese compromiso lleva directamente a la transformación, para bien, de la sociedad en que se vive.

¿Hacia dónde va la psicología social en América Latina? Hacia su consolidación como ciencia propia, en el sentido que al término propia da Fals Borda (1981); hacia la generación de teorías y métodos propios. Pero esto es algo que sólo podrá lograr en la medida en que se vuelque sobre la realidad concreta, en que se alimente interdisciplinariamente, en que logre establecer una rica y continua comunicación entre los países del área, multiplicando sus vías de expresión, las publicaciones. Lograrlo es tarea de todos, ya que la psicología social en América Latina para lograr el desarrollo, debe desarrollarse independientemente.

\section{Referencias}

DE CASTRo AGuIRRE, C.: Estereotipos nacionales en un grupo lationoamericano. Revista de Psicologia General y Aplicada, n. ${ }^{\circ}$ 92, pp. 235-267.

Díaz Guerrero, R.: Estudios de psicología del mexicano. México, Trillas, 1966.

- : Hacia una teoría bistórico-bio-psico-cultural del comportamiento bumano. México, Trillas, 1972.

Escovar, LUIS A.: Hacia un modelo psicológico-social del desarrollo. Boletín de la AVEPSO, vol. III, n. ${ }^{\circ} 1,1980$, pp. 1-6.

$-:$ Psicologia Social en América Latina. Diseño de un curso. Florida Internacional University, 1980.

- : El Psicólogo social y el desarrollo. Psicología, vol. IV, n. 3-4, pp. 367-378.

FINLEY, GoRDON y G. MARIN (edits).: Avances en psicologia social contemporánea. México, Trillas, 1979 (Incluye trabajos de cinco autores latinoamericanos).

Jurema, Ana C.: Psicología e Historia. Recife, Unicap, 1985.

LANe, Silvia y W. CODO (org.): Psicología social (O homem em movimiento). São Paulo, editora brasiliense, 1984 .

MARín, GERARDo (edit.): La Psicología social en Latinoamérica, vol. I, México, Trillas, 1975.

Vol. II, México, Trillas, 1981.

Martin-BARO, IgNACio: Acción e Ideología. Psicologia Social desde Centroamérica. San Salvador, UCA, 1983.

- Problemas de psicología social en América Latina. San Salvador, UCA, 1976.

- Psicologia social. San Salvador, UCA, 1984.

MONTERO, MARITZA: Ideología, alienación e identidad nacional. Caracas, EBUC, 1984.

- Estado actual de la Psicologia Social en Venezuela. Miami, XVI Congreso Interamericano de Psicología, 1976.

—- Para una psicología social histórica. Boletin de la AVEPSO, vol. I, n. ${ }^{\circ} 1,1978$.

- L L Psicología social en Venezuela. 1975-1985, Mérida, XXXV Convención de AsoVAC, 1985.

Pulido de Briceno, Mercedes: Piscología Social. Simposio. sobre Desarrollos recientes de la Psicología en Venezuela. Caracas, XX Congreso Interamericano de Psicología, 1985.

Riberio DE AlMEIDA, ANTONIO: Prolegomenos a uma História da Psicología Social Brasileira. Sintese Politica. Economia Social, n. ${ }^{\circ} 12$, sf.

RIBES IÑESTA, EMILIO: El papel de la psicologia en el cambio social. Psicología, vol. 3, n. ${ }^{\circ}$ 3-4, 1976, pp. 267-278.

RodRIGUES, AROLdo: Estudos em Psicologia Social. Petrópolis, Vozes, 1972. Hay una edición castellana en Trillas, 1975.

- - On the differential affects of some paramenters of balance. J. of Psychology, n. ${ }^{\circ} 61$, pp. 241-250, 1965.

_-: La psicología social problemas actuales y perspectivas para el futuro. La Psicología social en Latinoamérica (G. Marín, edit.), México, Trillas, 1981, pp. 17-35. 
RoDRigues, AROLDO y M. H. FerreIRA: Fontes de tendenciosidade cognitiva nas relaçóes interpessoais. Arquivos brasileiros de Psicotecnia. n. ${ }^{\circ} 2,1967$, pp. 9-21.

Salazar, J. M.: Bases psicológicas del nacionalismo. México, Trillas, 1983.

- : La Psicología social en Venezuela. Interamerican Psychologist, n. ${ }^{\circ} 60$, pp. 10-12, 1984. : The Use and Impact of Psychology. Int. J. of Psychology, 1984, pp. 113-122. - Vigencia y perspectivas de la Psicologia social. La Psicologia Social en Latinoamérica (edit. G. Marín). México, Trillas, 1981, pp. 36-47.

SAlazAr, S. M., M. MONTERo y otros: Psicología Social, Caracas, AVEPSO y Escuela de Psicologia, UCV, 1976. Hay edición en Edit. Trillas, México, desde 1978.

SÁNCHEZ, EUCLIDES y E. WIESENFELD: La psicología social aplicada: algunas consideraciones. Boletín de la AVEPSO, vol. V, n. ${ }^{\circ} 3,1982$, pp. 7-14.

VARELA, JACOBO: Can Social Psychology be applied?. Applying Social Psychology (edit. M. Deutsch y H. Hornstein). New York, Eribaum, 1975.

: Psicología social aplicada. La Psicología social en Latinoamérica. (edit. G. Marín), México, Trillas, 1975, pp.241-256.

-: Psychological solutions to social problems. New York, Academic Press, 1971.

: Tecnología social. La Psicología social en Latinoamérica. (edit. G. Marín). México, Trillas, 1981, pp. 139-165. 